ARTIGO ORIGINAL ORIGINAL ARTICLE

Palavras-chave:

diretrizes, análises de custo-efetividade, economia da saúde

\section{Diretriz metodológica para estudos de avaliação econômica de tecnologias em saúde no Brasil}

\author{
Methodological guidelines for economic evaluation \\ studies of health technologies in Brazil
}

Rodrigo Antonini Ribeiro 1,2,3, Jeruza Lavanholi Neyeloff1,4,5, Alexander Itria1,6, Vania Cristina Canuto Santos ${ }^{7}$, Cid Manso de Mello Vianna ${ }^{8}$, Everton Nunes da Silva ${ }^{9}$, Flávia Tavares Silva Elias ${ }^{10}$, Roberta Moreira Wichmann ${ }^{9,11}$, Kathiaja Miranda Souza , Luciane Nascimento Cruz ${ }^{1,12}$, André Luis Ferreira Azeredo-da-Silva1,5

DOI: 10.21115/JBES.v8.n3.p174-184

\section{RESUMO}

Objetivos: Resumir os principais pontos da Diretriz de Avaliação Econômica em Saúde (AES) do Ministério da Saúde. Métodos: As diretrizes para AES no Brasil foram desenvolvidas por intermédio de múltiplas rodadas de trabalho iterativas por grupo multidisciplinar de especialistas em economia da saúde e foram submetidas à consulta pública. Resultados: O problema deve ser definido por meio de uma questão de pesquisa estruturada. O estudo pode ser baseado em dados primários ou em modelagem, em que o primeiro aumenta a validade interna dos resultados e o segundo, a capacidade de generalização do estudo. Quando o trabalho for baseado em modelagem e focado em doença crônica, o modelo de Markov pode ser usualmente empregado, quando não houver necessidades que apontem para simulação de eventos discretos (como competição dos indivíduos por recursos escassos) ou modelos de transmissão dinâmica (em vacinação e/ou doenças infecciosas com alta transmissão entre indivíduos). O horizonte temporal preferencial é o de tempo de vida, e a taxa de desconto padrão é de 5\% para custo e efetividade. Os custos devem representar a perspectiva do Sistema Único de Saúde (SUS), podendo ser estimados por macrocusteio ou microcusteio. Sempre que possível, os resultados devem ser apresentados no formato de custo por ano de vida salvo ajustado para qualidade, para facilitar comparações com outros estudos. Análises de sensibilidade devem ser extensamente empregadas, de forma a avaliar o impacto da incerteza nos resultados produzidos. Conclusões: Espera-se que, com a padronização da metodologia proposta na Diretriz, a produção de AES no país tenha incremento na sua qualidade e reprodutibilidade.

\footnotetext{
ABSTRACT

Objectives: To summarize the main points from the Brazilian's Ministry of Health Economic Evaluations (HEE) guideline. Methods: The guideline was developed through multiple rounds of iterative work, conducted by a multidisciplinary team of specialists in health economics, and where submitted to public consultation. Results: The decision problem should be defined through a structured

Recebido em: 02/06/2016. Aprovado para publicação em: 08/08/2016

1. Instituto de Avaliação de Tecnologias em Saúde, Porto Alegre, RS, Brasil.

2. Programa de Pós-Graduação em Epidemiologia, Universidade Federal do Rio Grande do Sul (UFRGS), Porto Alegre, RS, Brasil.

3. Faculdade Meridional - IMED, Passo Fundo, RS, Brasil.

4. Unimed Porto Alegre, Porto Alegre, RS, Brasil.

5. Hospital de Clínicas de Porto Alegre, Porto Alegre, RS, Brasil,

6. Instituto de Patologia Tropical e Saúde Pública, Universidade Federal de Goiás (UFG), Goiânia, GO, Brasil.

7. Departamento de Gestão e Incorporação de Tecnologias, Secretaria de Ciência, Tecnologia e Insumos Estratégicos, Ministério da Saúde, Brasília, DF, Brasil.

8. Instituto de Medicina Social, Universidade do Estado do Rio de Janeiro (UERJ), Rio de Janeiro, RJ, Brasil.

9. Universidade de Brasília (UnB), Brasília, DF, Brasil.

10. Fundação Oswaldo Cruz (Fiocruz), Brasília, DF, Brasil.

11. Departamento de Ciência e Tecnologia, Secretaria de Ciência, Tecnologia e Insumos Estratégicos, Ministério da Saúde, Brasília, DF, Brasil. 12. Hospital Moinhos de Vento, Porto Alegre, RS, Brasil.

Nome da instituição onde o trabalho foi executado: Instituto de Avaliação de Tecnologias em Saúde.

Fontes externas de financiamento: Não houve.

Conflitos de interesse: $O$ s autores declaram não haver conflitos de interesse.

Autor correspondente: Rodrigo Antonini Ribeiro. Rua Quintino Bocaiuva, 1482, Porto Alegre, RS, Brasil. CEP: 90440-051. Telefone: +55 (51) 3013-1341. E-mail: rodrigo ribeiro@htanalyze.com
} 
research question. The study can be either primary data or model-based; in the first case, there is greater internal validity, while the second generates a superior generalizability. When the study is model-based and focused on a chronic disease, a Markov model can be usually employed, except for situations that points towards the need of a discrete event simulation (such as competition of individuals for scarce resources) or a dynamic transition model (for example, vaccination models and infectious diseases with high transmission rates between individuals). The preferred time horizon is the lifetime one, and the default discount rate is $5 \%$ for both costs and effectiveness. Costs should represent the Unified Health System (SUS) perspective and can be estimated through either gross-costing or micro-costing. Results should be presented as costs per quality adjusted life years (QALYS) whenever possible, to facilitate comparison with other studies. Sensitivity analyses should be widely employed, in order to evaluate the impact of uncertainty in the results produced by the model. Conclusions: It is expected that, with the methodological standardization proposed in this guideline, the HEE production in Brazil has gains in quality and reproducibility.

\section{Introdução}

A segunda década do século XXI está sendo caracterizada pela preocupação com a sustentabilidade de sistemas de saúde, em que a capacidade de se manter benefícios em saúde ao longo do tempo é determinante para a qualidade da atenção. Adicionalmente, nenhum país dispõe de recursos que permitam financiar intervenções terapêuticas ou diagnósticas que resultem em benefícios de magnitude pequena ou nula. A partir de 2008, países como Chile, Costa Rica, Croácia, Coreia do Sul e Uruguai implementaram agências voltadas à avaliação de tecnologias da saúde (ATS) (Stephens et al., 2012). Nesse período, o governo brasileiro ampliou sua capacidade técnica e executiva na área de ATS, o que ocorreu por meio da instituição de equipes especificamente designadas para esse fim no Departamento de Ciência e Tecnologia (DECIT) e da criação e fomento da Rede Brasileira de Avaliação de Tecnologias em Saúde (REBRATS), organização que congrega, além de representantes do Ministério da Saúde (MS), diversos grupos acadêmicos com foco no desenvolvimento de estudos de ATS.

Com a publicação em 2011 da Lei no 12.401, foi instituída a Comissão Nacional de Incorporação de Tecnologias no SUS (CONITEC), em substituição à comissão anterior, Comissão de Incorporação de Tecnologias do Ministério da Saúde (CITEC), para assessorar o MS na incorporação, exclusão ou alteração pelo Sistema Único de Saúde (SUS) de novos medicamentos, produtos e procedimentos. Segundo essa lei, a avaliação das tecnologias submetidas à CONITEC deve considerar, necessariamente, as evidências científicas sobre eficácia, efetividade, segurança e também análise econômica, com comparação dos benefícios e dos custos em relação às tecnologias já incorporadas. Assim, essa lei institucionalizou a ATS e os estudos de avaliação econômica como critérios necessários para a tomada de decisão sobre a incorporação tecnológica no SUS.

O objetivo deste artigo é sumarizar as principais orientações para a realização de avaliações econômicas em saúde na perspectiva do SUS, as quais foram publicadas na sua integralidade na Segunda Edição da Diretriz de Avaliação
Econômica (Ministério da Saúde, 2014). Este documento, que atualizou e ampliou os conceitos contidos na primeira edição da Diretriz, forma, com as recomendações para análise de impacto orçamentário (Ferreira-Da-Silva et al., 2012), o principal conjunto de recomendações para a formulação de estudos de avaliações econômicas em saúde que visem à incorporação de tecnologias no SUS. Ainda que o foco seja de fato o sistema público, vários conceitos aqui apresentados também podem ser úteis para avaliação de tecnologias na saúde suplementar. Os 19 pontos descritos a seguir resumem os capítulos correspondentes na Diretriz.

\section{Caracterização do problema}

Toda avaliação econômica deve estar orientada por uma questão de pesquisa bem definida, que responda ao acrônimo PICO (patient or population, intervention, comparison, outcome - em português, paciente ou população, intervenção, comparação e desfecho), à perspectiva da análise e ao horizonte de tempo. Essa questão de pesquisa delimitará o escopo do estudo e indicará o desenho da pesquisa e a técnica mais apropriada.

O Quadro 1 exemplifica a construção de uma pergunta com o detalhamento suficiente para um estudo de custo-efetividade. Além de específica, a questão de estudo deve ser relevante e orientada para as necessidades dos gestores envolvidos no processo de decisão. No caso do SUS, decisões relacionadas às tecnologias em saúde podem ser tomadas em vários níveis, que incluem desde o MS até os níveis estaduais e municipais.

\section{População em estudo}

A população-alvo deve ser descrita detalhadamente em termos de características clínicas, sociodemográficas, geográficas, e ainda quanto ao ambiente de inserção da tecnologia, padrões de tratamento e aderência à intervenção proposta. As fontes de dados para avaliação do desfecho em saúde também devem ser claramente apontadas. 
Quadro 1. Exemplo de Questão Genérica e Questão Completa Adequada

\begin{tabular}{|c|c|}
\hline \multicolumn{2}{|l|}{ Questão genérica: } \\
\hline \multicolumn{2}{|c|}{ Realizar mamografia como exame de rastreio é custo-efetivo? } \\
\hline \multicolumn{2}{|l|}{ Questão específica: } \\
\hline \multicolumn{2}{|c|}{$\begin{array}{l}\text { Qual é a razão de custo-utilidade incremental, considerando } \\
\text { o horizonte de tempo até o final da vida e a perspectiva } \\
\text { do SUS, de se realizar a mamografia de rastreamento do } \\
\text { câncer de mama em mulheres para a faixa etária de } 40 \text { a } \\
69 \text { anos, em vez de para a faixa de } 50 \text { a } 69 \text { anos atualmente } \\
\text { preconizada pelos protocolos do Ministério da Saúde? }\end{array}$} \\
\hline \multicolumn{2}{|l|}{ Construção da questão: } \\
\hline População em estudo & Mulheres com 40 anos ou mais \\
\hline Intervenção & Mamografia entre 40 e 69 anos \\
\hline Comparação & Mamografia entre 50 e 69 anos \\
\hline Desfecho & $\begin{array}{l}\text { Anos de vida ajustados } \\
\text { pela qualidade }\end{array}$ \\
\hline Perspectiva & $\begin{array}{l}\text { SUS, em âmbito federal } \\
\text { (Ministério da Saúde) }\end{array}$ \\
\hline Horizonte analítico & Até o fim da vida \\
\hline Cenário e contexto & $\begin{array}{l}\text { Estratégia de prevenção } \\
\text { secundária (rastreamento) } \\
\text { a ser realizada no âmbito } \\
\text { dos postos de saúde }\end{array}$ \\
\hline
\end{tabular}

\section{Desenho do estudo}

Os estudos de avaliação econômica possuem, de forma geral, duas opções de desenho: avaliação econômica baseada em dados primários ou estudo baseado em modelagem. Deve-se sempre explicitar em qual desses dois formatos a análise econômica é baseada. Para o primeiro caso, deve-se indicar o delineamento do estudo utilizado, bem como suas características metodológicas (se coleta de dados prospectiva ou retrospectiva, tamanho da amostra etc.) e possíveis vieses. No caso de uso de um modelo, é importante que ele seja adequadamente representativo do cenário clínico analisado, mas com a menor complexidade possível. Além de descrever o tipo de modelo adotado e seu desenho esquemático, devem-se indicar claramente as fontes de dados e os ajustes realizados às informações extraídas da literatura. Sugere-se que a abordagem utilizada em modelagem se baseie em dados empíricos e combine dados obtidos de estudos clínicos controlados (ou meta-análise destes) com elementos retirados de estudos observacionais, especialmente em relação às medidas de benefícios e à utilização dos recursos. Em ambos os casos, recomenda-se, sempre que possível, utilizar dados de efetividade em vez de eficácia, na medida em que os primeiros tendem a refletir a prática clínica em uso (Drummond et al., 1997).
Pode-se realizar uma avaliação econômica a partir de dados primários, em estudo desenhado exclusivamente para isso, ou conduzidos em paralelo com coortes ou ensaios clínicos planejados inicialmente para outros desfechos.

Os estudos baseados em dados primários apresentam a vantagem de maior validade interna, confiabilidade e consistência das informações utilizadas. No entanto, possuem a desvantagem de menor capacidade de generalização para além da amostra estudada nas estimativas de custo-efetividade, estando sujeitas a problemas decorrentes da diferença de objetivos entre uma avaliação econômica, que visa representar um cenário de uso real, e um ensaio clínico, que estuda indivíduos com formas mais homogêneas da doença e em ambientes experimentais controlados. Para uma visão mais aprofundada, sugere-se a leitura de diretriz recente que resume as boas práticas para a condução de análises aninhadas em ensaios clínicos randomizados (ECRs) (Ramsey et al., 2015).

Os modelos para análise de decisão são representações da complexidade do mundo real em uma forma simples e abrangente (Buxton et al., 1997). Em relação aos estudos baseados em dados primários, os modelos são capazes de representar de forma mais robusta o comportamento das relações de custo-efetividade adaptadas para diferentes circunstâncias clínicas e econômicas, integrando as melhores evidências de eficácia e de segurança às estimativas de custos mais apropriadas a uma determinada população-alvo, além de permitirem a realização de análises de sensibilidade que demonstram os limites das estimativas obtidas. Dessa forma, costumam ter maior validade externa do que os modelos baseados em dados primários, sendo os mais comumente encontrados na literatura. Os diferentes modelos utilizados são descritos no item 10.

\section{Tipos de análise}

As avaliações econômicas completas são classificadas de acordo com a medida do eixo de resultados, conforme a Tabela 1. Em uma análise de custo-minimização, as intervenções são consideradas equivalentes, e, portanto, procede-se apenas à comparação entre custos de cada uma. Análises de custo-efetividade (ACE) apresentam como resultado o custo por uma medida de desfecho da prática clínica (também chamadas de unidades naturais); exemplos clássicos são anos de sobrevida ou eventos finalísticos evitados (por exemplo: infarto agudo do miocárdio, novos casos de câncer). Nesse tipo de análise, deve-se evitar medição de custo por desfechos substitutos e mesmo intermediários, particularmente em pequenas unidades como "por mg/dL de LDL" ou "por mmHg de pressão arterial" reduzidos, devido à dificuldade em estimar o impacto clínico real desses dados. Já as análises de custo-utilidade (ACU) usam como desfecho "anos de vida 
Tabela 1. Análises econômicas completas

\begin{tabular}{|c|c|c|c|}
\hline Tipo de análise & $\begin{array}{l}\text { Medida de } \\
\text { custos }\end{array}$ & Medida de desfecho & $\begin{array}{l}\text { Medida de resultado } \\
\text { [ Fórmula ] }\end{array}$ \\
\hline Custo-minimização & $\begin{array}{l}\text { Valor } \\
\text { monetário }\end{array}$ & $\begin{array}{l}\text { Assume-se equivalência de desfechos } \\
\text { para as intervenções comparadas }\end{array}$ & $\begin{array}{l}\text { Apenas comparação de custos } \\
{\left[\left(C_{1}-C_{2}\right)\right]}\end{array}$ \\
\hline Custo-efetividade & $\begin{array}{l}\text { Valor } \\
\text { monetário }\end{array}$ & $\begin{array}{l}\text { Medida clínica } \\
\text { (anos de sobrevida, eventos evitados) }\end{array}$ & $\begin{array}{l}R \$ \text { / medida de desfecho ganho } \\
{\left[\left(C_{1}-C_{2}\right) /\left(E_{1}-E_{2}\right)\right]}\end{array}$ \\
\hline Custo-utilidade & $\begin{array}{l}\text { Valor } \\
\text { monetário }\end{array}$ & Anos de vida ajustados para qualidade & $\begin{array}{l}\mathrm{R} \$ \text { / QALY ganho } \\
{\left[\left(C_{1}-C_{2}\right) /\left(Q A L Y_{1}-Q A L Y_{2}\right)\right]}\end{array}$ \\
\hline Custo-benefício & $\begin{array}{l}\text { Valor } \\
\text { monetário }\end{array}$ & Convertida para valor monetário & $\begin{array}{l}\text { RS líquido } \\
{\left[\left(C_{\text {total }}-E_{\text {total }}\right)\right]} \\
\text { (será um valor positivo ou negativo) }\end{array}$ \\
\hline
\end{tabular}

C: custo; E: efetividade; QALY: quality adjusted life years (anos de vida ajustados para qualidade).

ajustados pela qualidade" (AVAQ ou QALY, quality adjusted life years), obtidos por meio de índices de utilidade de estados de saúde (utilities) para ponderar os anos de sobrevida. A grande vantagem dessa técnica é a possibilidade de comparação entre intervenções de diferentes áreas, sendo, portanto, a metodologia recomendada como de primeira escolha. Embasado em um estudo de custo-utilidade, o gestor pode averiguar se um programa para tratamento de hipertensão arterial é mais ou menos efetivo que a adoção de um novo teste diagnóstico para sífilis, por exemplo. Finalmente, análises de custo-benefício (ACB) convertem o eixo de consequências em valor monetário. A grande limitação desses estudos advém da dificuldade em valorar, em termos monetários, eventos de saúde, longevidade e qualidade de vida. Embora existam métodos para isso (capital humano, disposição a pagar), nenhum é isento de críticas, e há grande debate ético envolvido. Dentre os quatro tipos de análise, a preferencial para estudos conduzidos na perspectiva do SUS é a de custo-utilidade.

\section{Descrição das intervenções a serem comparadas}

Para que uma análise de decisão e custo-efetividade seja válida e útil na tomada de decisão, a tecnologia avaliada deve ser comparada a todas as estratégias relevantes para intervenção no problema de saúde que se está avaliando. As opções consideradas na avaliação econômica devem ser descritas em relação ao papel que desempenham nos cuidados com a saúde, sejam elas terapêuticas, diagnósticas, de rastreamento, preventivas ou para cuidados de apoio. Também devem ser classificadas quanto à classe de tecnologia em saúde da qual fazem parte: medicamentos e imunobiológicos, equipamentos médico-hospitalares, procedimentos clínicos ou cirúrgicos. Casos extremos hipotéticos podem ser considerados nos modelos para análise de decisão, como "nada fazer/ não tratar", desde que essa opção seja considerada relevante para o julgamento da eficiência relativa das diferentes alternativas. E, naturalmente, as estratégias de intervenção já utilizadas no SUS como tratamento padrão ou como opção disponibilizada pelo sistema de saúde devem ser consideradas na avaliação econômica. O conjunto completo das alternativas consideradas deve ser descrito em uma lista, e a inclusão ou não de cada opção na avaliação econômica realizada deve ser justificada.

\section{Perspectiva do estudo}

A definição da perspectiva do estudo é a base para a demarcação dos custos a serem considerados. Nas avaliações econômicas realizadas para o MS, a perspectiva do SUS como órgão comprador de serviços deve ser adotada. Isso significa que devem ser considerados todos os custos cobertos pelo sistema público de saúde, de acordo com a tabela de valores vigente.

De forma opcional, a avaliação econômica pela perspectiva da sociedade pode ser realizada. Nesse caso, devem ser incluídos todos os custos de produção do serviço/procedimento, do tempo perdido pelos pacientes e seus familiares, além dos custos relacionados à perda de produtividade e morte prematura.

\section{Horizonte temporal}

Ao escolher o horizonte temporal a ser considerado na avaliação econômica, deve-se levar em consideração a história natural do problema de saúde avaliado e os possíveis efeitos que a intervenção analisada poderá ter sobre ela. Essa escoIha deve ser capaz de capturar todas as consequências e os custos relevantes para os desfechos utilizados. Para doenças agudas e sem sequelas em longo prazo, recomenda-se adotar o menor período que permita avaliar os desfechos relevantes. Por outro lado, para doenças crônicas ou com desfechos em longo prazo, prioritariamente se deve adotar a expectativa de vida dos pacientes como horizonte temporal. Em todos os casos, as razões para a escolha de um determinado horizonte temporal deverão ser apresentadas. 


\section{Medidas de desfecho para análises econômicas}

O denominador da razão incremental que representa o resultado dos estudos de avaliação econômica completos corresponde à diferença em termos de desfechos em saúde. Estes podem ser classificados como primordiais (finalísticos) ou desfechos intermediários. Nas avaliações econômicas, preferencialmente devem ser utilizados os resultados finalísticos (por exemplo: infartos ou casos de câncer evitados, anos de vida ganhos). Se apenas resultados intermediários estiverem disponíveis, a relação entre estes e os resultados finalísticos deve ser estimada com base na literatura, devendo ser claramente mencionado e justificado no estudo e testado na análise de sensibilidade. Em todos os casos, uma estimativa da qualidade de vida deverá ser integrada às estimativas dos desfechos finalísticos.

A importância da avaliação de qualidade de vida vem aumentando nos últimos anos, principalmente por ser um enfoque que valoriza a percepção do paciente. A utilidade (utility), que faz uma ponderação do tempo de vida ganho para a qualidade desta, deve ser utilizada principalmente em doenças crônicas e situações em que existam certas sequelas ou incapacidades secundárias, nas quais o impacto da tecnologia não se limita apenas a mudanças nas taxas de morbimortalidade, mas sim ao bem-estar subjetivo dos indivíduos.

Em termos de aferição do efeito na qualidade de vida, a medida escolhida para uma análise de custo-utilidade deve ser genérica a fim de possibilitar comparações entre programas, e ela deve estar traduzida e validada para cada contexto em que o estudo está sendo realizado. Exemplos de medidas de utilidade genéricas são o Standard Gamble, EQ-5D e SF-6D. O Standard Gamble e Time-Trade-off não demandam um processo formal de tradução e validação, podendo ser aplicados diretamente nos pacientes para avaliar valores para estados de saúde. Por outro lado, os instrumentos compostos por questionários como EQ-5D e SF-6D necessitam ser traduzidos e adaptados culturalmente; tal processo já foi realizado no Brasil recentemente (Cruz et al., 2011, Santos et al., 2015). Portanto, para análises econômicas realizadas no Brasil, dados primários em qualidade de vida poderão ser coletados por meio dos instrumentos Standard Gamble, Time Trade-Off, SF-6D ou EQ-5D.

Nas situações em que não houver dados primários de qualidade de vida, os pesquisadores necessitarão utilizar informações da literatura. Nesse caso, devem estar atentos a dois aspectos importantes: priorizar índices de utilidade coletados em populações semelhantes à população considerada no estudo e agregar apenas índices de utilidade que foram aferidos pelo mesmo instrumento (por exemplo, Standard Gamble ou EQ-5D), uma vez que a literatura vem demonstrando que os valores de utilidade não são os mesmos para um mesmo estado de saúde quando são gerados por instrumentos diferentes.

Em algumas situações em análises de custo-efetividade, os desfechos são medidos em unidades naturais. Exemplos incluem: número de casos de determinada doença curados, anos de vida ganhos, hospitalizações evitadas etc. A limitação dessa modalidade de análise é que a unidade mais específica de desfecho não permite comparações entre diferentes estratégias e não incorpora as preferências dos indivíduos para esses desfechos.

Para obter-se os AVAQ, deve-se multiplicar o resultado obtido em um dos escores de utilidade (como o SF-6D ou o EQ-5D) pelos anos de vida passados em determinado estado de saúde. Como os índices de utilidade são medidos em escalas entre 0 e 1, em que 1 representa saúde perfeita, para simplificação, os AVAQ podem ser medidos em "anos em saúde perfeita" (um ano em perfeita saúde = 1 AVAQ, meio ano em perfeita saúde = 0,5 AVAQ).

Na identificação de fontes de informações para os desfechos em saúde, as revisões sistemáticas de ensaios clínicos randomizados são o método mais robusto para avaliação da eficácia das intervenções.

Eventos adversos associados com a intervenção devem ser incluídos e valorados nas avaliações quando eles forem clínica e/ou economicamente importantes e baseados em diferenças significativas entre a intervenção estudada e suas alternativas.

\section{Quantificação e custeio de recursos}

A estimativa dos custos implica três etapas: (1) definição da perspectiva da análise e dos custos relevantes à avaliação, (2) mensuração dos recursos usados, e (3) valoração dos recursos. Quando a perspectiva de análise for do SUS, devem ser incluídos todos os custos diretos envolvidos no cuidado prestado pelo sistema de saúde. Custos indiretos somente seriam considerados em uma análise se baseados na perspectiva social. Custos intangíveis não devem ser incluídos nas análises realizadas para o SUS, pois sua mensuração e valoração são controversas.

As estimativas de custos devem ser apresentadas para determinado ano, sendo necessário o ajuste pela passagem do tempo: no caso de custos que serão incorridos no futuro, deve-se aplicar uma taxa de desconto para trazê-los a valor presente; no caso de custos realizados no passado, deve-se corrigi-los pela taxa de inflação (como o Índice Nacional de Preços ao Consumidor Amplo - IPCA), para trazê- los a valor real.

No caso de modelos de avaliação econômica baseados em estados de saúde, a utilização de recursos em cada estado de saúde deve ser representada em uma função de produção que mostre os custos gerados pela utilização 
de recursos nas estratégias analisadas. Sob a perspectiva do SUS, a análise pode ser feita considerando o SUS como um comprador de serviços de saúde ou, alternativamente, como uma ou mais unidades prestadoras de serviços de saúde (devendo a escolha entre essas alternativas ser explicitada). Quando a perspectiva adotada for a primeira, os valores de reembolso pagos pelo SUS para os diferentes itens devem ser utilizados como medida de valoração dos custos. No segundo caso, os itens de custos envolvidos devem ser identificados e valorados, e a metodologia deve ser detalhada no estudo.

Quanto à valoração dos recursos, tanto as abordagens de microcusteio quanto a de macrocusteio podem ser utilizadas (ou uma composição de ambas). No microcusteio, também conhecido como método bottom-up ("de baixo para cima"), identificam-se detalhadamente os itens geradores de custos por revisão de prontuários ou questionários prospectivos. No macrocusteio, ou método top-down ("de cima para baixo"), estima-se um custo médio aproximado de cada item gerador de custo a partir de bases de dados como o DATASUS e outros registros administrativos de hospitais ou de outros componentes do sistema de saúde. Para que isso seja possível, a base de dados utilizada deve conter as informações de custo total utilizado para uma determinada jurisdição e da quantidade de itens que foram utilizados em um determinado intervalo de tempo. Enquanto com o microcusteio há maior precisão dos dados, no macrocusteio há maior facilidade de reprodução dos estudos para outros lugares.

Geralmente, não existe uma única fonte de dados por meio da qual podem ser obtidas todas as informações relevantes sobre utilização de recursos e seus custos, havendo necessidade da combinação de diversas fontes de informação. No âmbito das avaliações econômicas realizadas sob a perspectiva do SUS, o sítio de internet DATASUS contém as principais fontes de informação para obtenção de dados de custeio. Nessa fonte, podem ser relevantes os sistemas de informações de mortalidade (SIM), de dados hospitalares (SIH-SUS) e ambulatoriais (SIA-SUS). Outras fontes de informação relevantes incluem o banco de preços em saúde (BPS), que armazena os valores pagos por instituições credenciadas ao SUS no momento da compra de bens, além de bancos de dados hospitalares, registros médicos, literatura científica e consulta a painel de especialistas, que deve ser utilizado como último recurso, na ausência de fontes mais objetivas de informação. As fontes das informações de custeio devem ser explicitamente relatadas na avaliação econômica realizada.

\section{Modelagem}

Modelos são representações da realidade, e, no contexto de análise econômica, servem ao propósito de informar sobre decisões em saúde e alocação de recursos. Os modelos são ferramentas analíticas com as quais, após ser criado o cenário da doença em avaliação, é possível comparar diferentes estratégias em saúde, de forma a definir seus resultados e custos. Quando não há a disponibilidade de uma análise econômica aninhada a um ensaio clínico, a modelagem é a abordagem a ser utilizada para fornecer respostas para tomadores de decisão na área da saúde.

As recomendações sobre modelagem propostas aqui seguem usualmente o que foi sugerido em uma série de publicações conjuntas de duas entidades da área de estudos econômicos em saúde (Briggs et al., 2012, Caro et al., 2012, Eddy et al., 2012, Karnon et al., 2012, Pitman et al., 2012, Roberts et al., 2012, Siebert et al., 2012), com adaptações ao SUS e à realidade brasileira, quando necessário.

\section{Idealização e concepção do problema}

Antes de iniciar a concepção do modelo, é necessário compreender o problema, ou seja, transformar o conhecimento sobre o processo de saúde em questão em uma representação prática do problema, que possa ser representada por meio de um modelo.

Os pesquisadores envolvidos na criação do modelo deveriam receber as opiniões de experts da área do problema clínico e de tomadores de decisão, para assegurar que o modelo represente adequadamente o processo clínico e ao mesmo tempo contemple o problema decisório.

Após essa fase inicial, uma declaração clara de qual é o problema de decisão, o objetivo do modelo e seu escopo deve ser realizada. Isso deve englobar o espectro da doença, a perspectiva da análise, a população-alvo, as intervenções consideradas, os desfechos e o horizonte temporal.

\section{Idealização e concepção do modelo}

Após as definições listadas no item anterior, inicia-se o processo de criação do modelo, no qual é necessário assegurar que todos os pressupostos definidos sejam de fato incorporados em sua estrutura. O primeiro passo na concepção do modelo é definir o seu tipo, sendo os mais comuns: árvores de decisão, modelos de estados transicionais (também chamados de modelos de Markov), simulações de eventos discretos (SED) e modelos de transmissão dinâmica. As árvores de decisão têm seu uso limitado, sendo adequadas apenas para situações com horizonte temporal bastante curto, especialmente nas doenças agudas, em que não costuma haver repetição de eventos. Os modelos de Markov (que são os mais comumente vistos na literatura), por outro lado, são interessantes para horizontes de tempo maiores e quando as probabilidades no modelo variam ao longo do tempo. A simulação de eventos discretos (SED) é útil quando se deseja representar sujeitos de forma individual, especialmente quando existe 
interação entre eles e/ou quando há escassez de recursos (como em situações de transplante de órgãos). Finalmente, modelos de transmissão dinâmica (ou simplesmente modelos dinâmicos) são úteis quando interações entre grupos têm impacto nos resultados, como no caso de vacinações.

Para a escolha do tipo de modelo, a equipe envolvida em sua concepção deve responder às perguntas a seguir. De acordo com essas respostas, é dado o direcionamento para o modelo adequado, conforme resumido na Figura 1.

\section{Qual o horizonte temporal apropriado?}

Caso o horizonte não seja muito curto, as árvores de decisão simples não são recomendadas. Nas demais situações, outros tipos de modelo (mais comumente Markov ou SED) devem ser utilizados.

O modelo funcionará adequadamente com grupos de indivíduos ou é necessário representá-los individualmente?

As árvores de decisão e modelos de Markov convencionais não representam sujeitos de forma individual, e sim os tratam como uma fração de uma coorte homogênea. Por outro lado, modelos de Markov com microssimulação e SED representam cada paciente individualmente. A abordagem individual permite maior riqueza de detalhes no modelo, especialmente no tocante a características clínicas e a eventos sofridos interferirem na probabilidade de novos eventos.

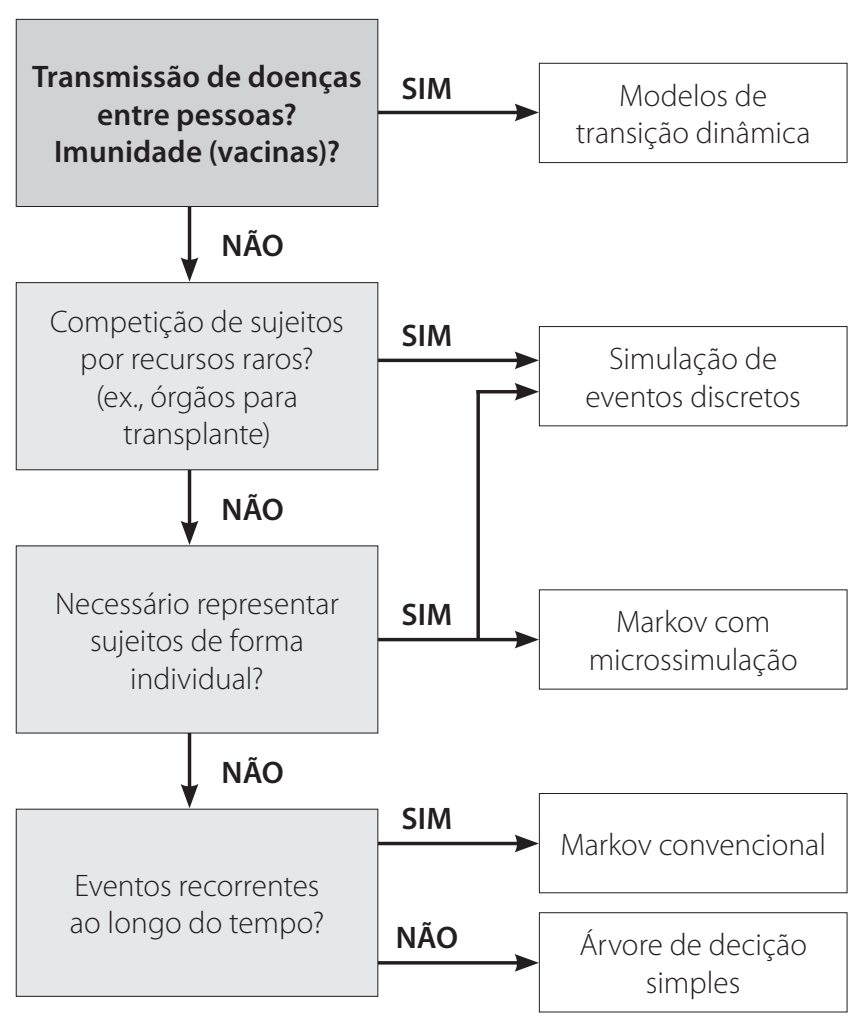

Figura 1. Fluxograma para escolha do modelo adequado.
Por outro lado, modelos que lidam com grupos são mais facilmente programáveis, e o tempo computacional para as análises é substancialmente menor. O efeito de eventos prévios pode ser modelado utilizando Markov convencional, por meio do incremento de número de estados. Porém, algumas situações fazem o número de tais estados de saúde se tornar exponencialmente grande, o que pode ser prejudicial ao modelo, sugerindo utilização de abordagens individuais (SED ou Markov com microssimulação).

\section{Caso represente indivíduos, há necessidade de contemplar interações entre os indivíduos, ou entre estes e componentes do modelo (como no caso de restrições na disponibilidade de recursos)?}

Quando a intervenção modelada tem efeito na transmissão de doenças, como em algumas situações de doenças infecciosas, o modelo deveria permitir interação entre os indivíduos, com a escolha recaindo em modelos dinâmicos ou SED. O mesmo se aplica a modelos em cenários com recursos limitados, como no caso de transplantes.

\section{Eventos podem acontecer mais de uma vez?}

As árvores de decisão simples só têm utilidade na modelagem de eventos que ocorrem apenas uma vez. Nas demais situações, outros tipos de modelos devem ser buscados.

\section{O tempo deve ser representado de forma contínua ou discreta?}

O modelo de Markov trata o tempo de forma discreta, isto é, há intervalos fixos de tempo em que eventos só podem ocorrer uma vez durante cada intervalo. Caso os eventos sejam frequentes, o modelo de Markov pode ter sua duração de ciclo diminuída. Modelos como SED, por outro lado, tratam o tempo de forma contínua, de maneira que eventos podem acontecer em qualquer instante de tempo, com período de eventual recorrência de eventos também bastante flexível. Caso isso seja desejável no modelo, esse tipo de abordagem deve ser escolhido.

Não existe apenas uma possibilidade de modelo para cada situação. As principais situações que apontam para a utilização específica de um modelo são:

- Em cenários de doenças infecciosas em que seja importante representar a interação entre os sujeitos e seu efeito em transmissão e imunidade (quando se deve lançar mão de modelos dinâmicos);

- Em situações de recursos com disponibilidade limitada e disputa entre os indivíduos por estes, em que a SED é o método de escolha.

Nas demais doenças crônicas, usualmente é possível utilizar Markov tradicional (isto é, com avaliação de coortes), Markov com microssimulação ou SED. O principal atrativo do Markov tradicional é sua ampla difusão (e consequente 
aceitabilidade pelo público leitor), simplicidade de montagem e facilidade para realizar análises de sensibilidade. Caso o número de estados se torne muito grande para acomodar todas as combinações de características clínicas desejadas, pode-se lançar mão de uma microssimulação. A SED pode, em geral, ser usada em situações em que ambos os tipos de Markov (coortes e microssimulação) são opções válidas; porém, tendo em vista sua maior necessidade de parâmetros a serem estimados, maior dificuldade computacional e menor familiaridade do público leitor, ela deve ser utilizada quando seu maior refinamento metodológico realmente se traduzir em ganhos na acurácia do modelo. A descrição detalhada de cada tipo de modelo pode ser encontrada no texto completo da Diretriz (Ministério da Saúde, 2014).

\section{Transparência e validação}

A principal função de modelos é fornecer informações para a tomada de decisão. Para tanto, é fundamental que eles se mostrem confiáveis. Dentre as principais maneiras de aumentar a credibilidade do modelo, deve-se atentar para os quesitos de transparência e validação:

- Transparência: diz respeito a descrever claramente a estrutura do modelo, suas equações, valores de parâmetros (e suas fontes), pressupostos, limitações e fontes de financiamento.

- Validação: está relacionada à verificação do modelo, de forma a demonstrar que os resultados projetados estão de acordo com o que é visto na realidade. Inclui quatro possíveis etapas: validade de face (face validity), verificação, validação cruzada (cross validation) e validação (Eddy et al., 2012).

\section{Taxa de desconto}

Para realizar uma comparação direta entre custos e desfechos em diferentes momentos no período de tempo estudado, é necessária a aplicação de taxas de desconto. Essas taxas ajustam a expectativa de futuros valores financeiros e de consequências para a saúde para estimativas de valores presentes, dentro da teoria de preferência temporal, que assume que não é possível comparar diretamente cifras presentes e futuras sem que seja realizado um desconto dos valores futuros. Desse modo, quando o horizonte temporal de análise for superior a um ano, tanto custos como resultados em saúde que ocorrem no futuro devem ser descontados ao seu valor no momento presente, usando-se uma taxa de desconto padrão. Para aumentar a comparabilidade dos estudos, sugere-se padronizar as taxas de desconto de custos e resultados em saúde em 5\% ao ano. Recomenda-se usar, na análise de sensibilidade, diferentes taxas de desconto (0\% e 10\%), para se determinar em que extensão a seleção arbitrária da taxa afetou a conclusão do estudo.

\section{Resultados}

A maioria das avaliações econômicas em saúde contempla tecnologias concorrentes (ou mutuamente excludentes, isto é, somente uma das estratégias pode ser adotada). Após o cálculo dos custos e efetividades das estratégias, elas devem ser ordenadas, iniciando-se com a mais barata até a mais cara. Após o ordenamento de acordo com os custos, são calculadas as razões de custo-efetividade incrementais (RCEl), as quais são obtidas por meio da divisão da diferença de custos das alternativas pela diferença das suas efetividades.

$$
\text { Razão de Custo-Efetividade Incremental }(R C E I)=\frac{C_{1}-C_{2}}{E_{1}-E_{2}}
$$

A RCEl expressa um resumo dos resultados de uma avaliação comparativa de diferentes estratégias de cuidados à saúde, sendo o principal resultado de uma análise econômica. Em caso de análises econômicas com múltiplas opções, usualmente as RCEI são calculadas em relação à estratégia com custo imediatamente mais barato, isto é, o procedimento padrão não é realizar cálculo de RCEls de cada estratégia utilizando sempre a estratégia mais barata como base de comparação. Isso se deve ao modo de interpretação das RCEI de acordo com valores de limiar de disposição a pagar, conforme comentado mais adiante.

O seguinte procedimento deve ser seguido na apresentação dos resultados:

- Elencar os programas do menor custo para o maior;

- Eliminar todas as estratégias que são dominadas (custos maiores e efetividade igual ou menor) por qualquer uma das outras;

- Para as estratégias não dominadas, a interpretação das RCEls é feita, de forma sucessiva, à luz do valor limiar de disposição a pagar (willingness to pay threshold). Imagine a situação a seguir (valores hipotéticos).

Caso o valor de disposição a pagar por um ano de vida fosse de R\$ 20.000 (valor hipotético, apenas para fins de exemplo), o raciocínio seria o seguinte (dados mostrados na Tabela 2):

- Para implantar a estratégia AAS, em comparação com nenhum tratamento, a RCEl encontra-se abaixo do limiar de disposição a pagar, então, ela seria considerada economicamente atrativa;

- Para implementar a estratégia AAS + clopidogrel, a RCEl em relação à estratégia de AAS isolado também se situa abaixo do valor de disposição a pagar, sendo considerada também economicamente atrativa, substituindo o AAS isolado como opção mais interessante do ponto de vista farmacoeconômico; 
- Na comparação entre AAS + nova medicação e AAS + clopidogrel, a RCEl entre as tecnologias se situa acima do que foi definido como aceitável em termos de disposição a pagar, sendo então a estratégia AAS + nova medicação rejeitada do ponto de vista econômico. Então, a conclusão do cenário é que, considerando o limiar proposto de disposição a pagar, a tecnologia mais custo-efetiva é AAS + clopidogrel.

Tabela 2. Exemplo de resultados de estudo de custo-efetividade

\begin{tabular}{lccc} 
Estratégia & Custo (R\$) & $\begin{array}{c}\text { Efetividade } \\
\text { (anos de vida) }\end{array}$ & $\begin{array}{c}\text { RCEI (R\$ por } \\
\text { anos de vida) }\end{array}$ \\
\hline $\begin{array}{l}\text { Nenhum } \\
\text { tratamento }\end{array}$ & 1.000 & 3 & - \\
\hline AAS & 4.500 & 3,6 & 5.833 \\
\hline $\begin{array}{l}\text { AAS + } \\
\text { Clopidogrel }\end{array}$ & 9.000 & 3,9 & 15.000 \\
\hline $\begin{array}{l}\text { AAS + nova } \\
\text { medicação }\end{array}$ & 15.000 & 4,1 & 30.000 \\
\hline
\end{tabular}

AAS: ácido acetilsalicílico.

\section{Análise de sensibilidade}

Avaliações econômicas em saúde são realizadas em condições de incerteza. O tipo de incerteza mais frequentemente citado é a relacionada aos valores das variáveis utilizadas no modelo (incerteza de parâmetros), os quais usualmente são oriundos de amostras e estão associados a uma margem de erro em relação ao real valor do parâmetro populacional. Existem ainda dois outros tipos de incerteza: a relacionada à estrutura do modelo (isto é, modo de construção da árvore de decisão ou do modelo de Markov, por exemplo) e a relacionada a aspectos metodológicos, que diz respeito a decisões sobre abordagens analíticas utilizadas (por exemplo, incorporação ou não de custos indiretos) (Jain et al., 2011).

A análise de sensibilidade serve a dois propósitos: refletir a incerteza associada aos resultados do modelo e auxiliar na definição de qual o valor da coleta de informações adicionais que possam melhor informar a tomada de decisão.

Uma análise de sensibilidade para avaliar a incerteza relacionada à estimava de parâmetros consiste de três estágios: identificação dos parâmetros que são objetos de questionamento, escolha de uma faixa plausível de variação dos fatores relacionados à incerteza e apresentação dos diferentes resultados decorrentes da variação dos parâmetros selecionados.

As análises univariadas - usualmente conduzidas de forma determinística - devem ser realizadas obrigatoriamente nas estimativas de parâmetros considerados como mais importantes do modelo. Limites plausíveis de variação dos parâmetros deverão ser definidos e justificados. Esses limites deverão refletir a escala total da variabilidade e da incer- teza que é relevante e apropriada para cada modelo. Tais limites usualmente serão determinados a partir da revisão da literatura; mas, quando esta for escassa, poderá ser feita pela consulta a especialistas ou testando cenários extremos. Quando os dados provêm de amostras, e esta amostra tiver uma distribuição de valores - usualmente a normal - pode-se usar como variação os limites do intervalo de confiança da estimativa. Outra abordagem seria, caso os dados vierem de um conjunto de estudos, utilizar o menor e o maior valor encontrados nesse conjunto. Finalmente, também é possível realizar variações arbitrárias. Por exemplo, se for necessário utilizar um valor de stent em um modelo, e o SUS realizar o pagamento de um valor fixo por ele, não há uma variação ou incerteza implícita no parâmetro. Porém, pode ser interessante saber o que aconteceriam com os resultados caso o valor aumentasse ou decrescesse em $25 \%$ ou $50 \%$. Essa análise de sensibilidade poderia ser empregada; porém, é importante notar que ela não está avaliando a incerteza, e sim a sensibilidade dos resultados a uma variação que poderia ocorrer em algum momento no futuro.

O relato da análise univariada pode ser feito de forma textual, descrevendo-se o intervalo da RCEl correspondente à variação do parâmetro, e também de forma gráfica, por meio do Diagrama de Tornado. Outra maneira interessante de apresentar análises univariadas é a análise de limiar (threshold analysis). Nela, a abordagem é inversa: em vez de se definir a faixa de variação do parâmetro, é calculado qual o valor do parâmetro que seria necessário para que a tecnologia se tornasse custo-efetiva, de acordo com um limiar preestabelecido de disposição a pagar.

Para a incorporação tecnológica no SUS, recomenda-se, além de análises univariadas, que sejam conduzidas análises multivariadas, nas quais são oscilados vários parâmetros simultaneamente, de forma a avaliar a robustez global dos resultados. Elas devem ser feitas de forma probabilística (simulação de Monte Carlo de segunda ordem), em que a variação nos parâmetros é feita de forma estocástica. A variação dos parâmetros é realizada na forma de distribuições de probabilidade, sendo as mais utilizadas: lognormal para o risco relativo, beta para probabilidades e valores de utilidade, e gama ou lognormal para custos.

As análises probabilísticas são realizadas com 1.000 ou mais simulações. A apresentação de dados deve ser feita por meio de diagrama de dispersão ou de curvas de aceitabilidade de custo-efetividade.

\section{Generalização dos resultados}

Generalização ou transferência dos resultados das avaliações econômicas refere-se à extensão com que os resultados de um estudo baseado em medidas de uma população particular de pacientes e/ou de um contexto específico podem ser 
aplicados ou extrapolados para outra população e/ou contexto diferente (Sculpher et al., 2004; Willke, 2003).

A generalização dos resultados das avaliações econômicas de intervenções em saúde apresenta limitações e problemas porque, muitas vezes, tanto o desenho da intervenção ou programa de saúde quanto, especialmente, seus custos podem variar significativamente.

Quando o estudo utilizar dados clínicos e epidemiológicos de outro país, os autores devem demonstrar que esses dados foram transferidos com suficiente plausibilidade para o contexto brasileiro. Porém, as diferenças de custos em saúde entre diferentes países (tanto em valores absolutos quanto relativos) são grandes o suficiente para que usualmente seja proibitivo o uso de dados de estudos econômicos internacionais para o cenário brasileiro, mesmo que se tente fazer alguma adaptação de custos. Dessa forma, não se recomenda utilização de dados econômicos de outros países.

\section{Limitações do estudo}

Todas as limitações do estudo devem ser relacionadas e discutidas, incluindo os problemas metodológicos, a validade das hipóteses feitas, a confiabilidade dos dados utilizados para estimativas de parâmetros e o uso de modelos na execução dos estudos de custo-efetividade.

Tendo em vista que modelos econômicos usualmente são dependentes de um grande número de estimativas de parâmetros, os quais frequentemente são coletados da literatura, a limitação dos resultados da análise econômica será diretamente proporcional às possíveis limitações (de qualidade e capacidade de transferência) dos dados obtidos.

\section{Aspectos éticos e administrativos}

Todos os estudos envolvendo seres humanos devem apresentar a aprovação de um comitê de ética em pesquisa institucional aprovado pela Comissão Nacional de Ética em Pesquisa, segundo a Resolução 466/12 do Conselho Nacional de Saúde/MS. Essa aprovação pela Comissão de Ética em Pesquisa institucional é obrigatória sempre que houver o levantamento de dados primários envolvendo pacientes.

\section{Conclusões e recomendações do estudo}

O estudo deve conter todos os elementos referentes a metodologia adotada, fontes utilizadas, relevância das informações coletadas e acurácia dos cálculos efetuados.

O produto final dos estudos de avaliação econômica completos é a estimativa de uma RCEl de uma nova alternativa tecnológica em relação à alternativa padrão. Em tese, esse valor poderia ser contraposto a um valor limiar de disposição a pagar, abaixo do qual a tecnologia seria considerada custo-efetiva. No entanto, as organizações produtoras e avaliadoras dos estudos econômicos em saúde no Brasil ainda não definiram tal valor limiar.

Por fim, tópicos para pesquisas futuras podem ser apontados a partir da identificação de parâmetros influentes nas análises de sensibilidade para os quais haja uma escassez de fontes confiáveis para estimativa. A identificação dessas áreas deficientes em informações originais aplicáveis ao cenário brasileiro poderá auxiliar na priorização de áreas de pesquisa.

\section{Conflitos de interesse/fontes de financiamento}

Todos os autores que participaram do estudo devem ser identificados e citados, com suas afiliações institucionais. As fontes de financiamento devem ser claramente mencionadas, assim como seu papel na concepção e condução do estudo. Todos os autores devem informar se existe algum tipo de conflito de interesse que possa estar influenciando os resultados obtidos.

\section{Limiar de disposição a pagar}

Depois de encerrar a análise econômica de determinada tecnologia, cabe ao gestor tomar a decisão de implementá-la ou não. Para compreender o conceito de limiar de disposição a pagar (LDP, em inglês willingness to pay [WTP] threshold), é preciso ter em mente que o objetivo do sistema é maximizar o benefício provido dentro de uma determinada restrição orçamentária - caso não houvesse limites para o investimento, bastaria implementar todas as intervenções com efetividade comprovada. Assim, estabelecer o valor de uma tecnologia exige uma avaliação sobre se a saúde adicional prevista para ser adquirida com a sua utilização excede as perdas de saúde com outros tratamentos que serão deslocados pelo seu custo adicional; essa comparação é adequadamente representada pela comparação da razão de custo-efetividade incremental (RCEI) com um limite definido para ela. O LDP deve representar a alternativa menos custo-efetiva incorporada ao sistema (Claxton et al., 2008).

A adoção de um LDP auxilia o objetivo de maximizar o ganho social do sistema (Johannesson e O'Conor, 1997). Idealmente, o valor do limiar deve advir de estudos sobre valor em saúde e de uma compreensão global da sociedade sobre o tema. Em um sistema que passa a considerar análises econômicas, limiares passarão a existir, sejam eles aparentes ou não; a adoção formal de um limiar promove uniformidade nas decisões sobre quais tecnologias serão adotadas (Giacomini, 2007).

Alguns países do mundo têm definições bastante claras do seu valor de LDP, como o Reino Unido, a Austrália, a Nova Zelândia, o Canadá e os Países Baixos (Cleemput et al., 2008, National Institute for Clinical Excellence). 
No Brasil, ainda não há pesquisa dedicada ao tema de valor em saúde e LDP consolidada, nem metodologia escolhida para determinação de tal limiar. Isso provavelmente é compatível com o momento ainda jovem do país em análises econômicas em saúde, e espera-se que esse panorama mude com avanço e maturidade das áreas envolvidas em ATS.

\section{Considerações finais}

Foram apresentados neste artigo os elementos fundamentais para a realização de estudos de avaliação econômica na área da saúde conforme as recomendações oficiais do Ministério da Saúde do Brasil. Estas foram elaboradas em conjunto por profissionais da área acadêmica e do Ministério da Saúde, combinando as visões desses dois atores em um único documento. O planejamento de estudos de avaliação econômica seguindo as etapas sugeridas funcionará como um ponto de partida confiável para a maioria dos estudos. A utilização de análises econômicas em saúde, quando adequadamente realizadas, é um ponto fundamental para a utilização racional de recursos na área da saúde. Os desafios para implementação da diretriz estão relacionados a pouca tradição e a ainda insuficiente capacidade instalada no país, tanto na academia como em órgãos gestores responsáveis por priorização e incorporação de intervenções em saúde. A qualificação de ambas as partes, especialmente em temáticas de menor difusão no país, como modelos de transição dinâmica e simulação de eventos discretos, é fundamental para a adequada aplicação da diretriz.

\section{Referências bibliográficas}

Briggs AH, Weinstein MC, Fenwick EA, Karnon J, Sculpher MJ, Paltiel AD. Model parameter estimation and uncertainty: a report of the ISPORSMDM Modeling Good Research Practices Task Force--6. Value Health. 2012;15(6):835-42.

Buxton MJ, Drummond MF, Van Hout BA, Prince RL, Sheldon TA, Szucs T, et al. Modelling in economic evaluation: an unavoidable fact of life. Health Econ. 1997;6(3):217-27.

Caro JJ, Briggs AH, Siebert U, Kuntz KM. Modeling good research practices-overview: a report of the ISPOR-SMDM Modeling Good Research Practices Task Force--1. Value Health. 2012;15(6):796-803.

Claxton K, Briggs A, Buxton MJ, Culyer AJ, McCabe C, Walker S, et al. Value based pricing for NHS drugs: an opportunity not to be missed? BMJ. 2008;336(7638):251-4.

Cleemput I, Neyt M, Thiry N, De Laet C, Leys M. Threshold values for costeffectiveness in health care. In: Belgian Health Care Knowledge Centre (KCE), editor. Brussels; 2008.
Cruz LN, Camey SA, Hoffmann JF, Rowen D, Brazier JE, Fleck MP, et al. Estimating the SF-6D value set for a population-based sample of Brazilians. Value Health. 2011;14(5 Suppl 1):S108-14.

Drummond MF, Richardson WS, O'Brien BJ, Levine M, Heyland D. Users' guides to the medical literature. XIII. How to use an article on economic analysis of clinical practice. A. Are the results of the study valid? Evidence-Based Medicine Working Group. JAMA. 1997;277(19):1552-7.

Eddy DM, Hollingworth W, Caro JJ, Tsevat J, McDonald KM, Wong JB. Model transparency and validation: a report of the ISPOR-SMDM Modeling Good Research Practices Task Force--7. Value Health. 2012;15(6):843-50.

Ferreira-Da-Silva AL, Ribeiro RA, Santos VC, Elias FT, d'Oliveira AL, Polanczyk CA. [Guidelines for budget impact analysis of health technologies in Brazil]. Cad Saude Publica. 2012;28(7):1223-38.

Giacomini M. How good is good enough? Standards in policy decisions to cover new health technologies. Healthc Policy. 2007;3(2):91-101.

Jain R, Grabner M, Onukwugha E. Sensitivity analysis in cost-effectiveness studies: from guidelines to practice. Pharmacoeconomics. 2011;29(4):297-314.

Johannesson M, O'Conor RM. Cost-utility analysis from a societal perspective. Health Policy. 1997;39(3):241-53.

Karnon J, Stahl J, Brennan A, Caro JJ, Mar J, Moller J. Modeling using discrete event simulation: a report of the ISPOR-SMDM Modeling Good Research Practices Task Force--4. Value Health. 2012;15(6):821-7.

Ministério da Saúde. Diretrizes Metodológicas: Diretriz de Avaliação Econômica. 2 ed. Brasília; 2014.

National Institute for Clinical Excellence N. Guide to the Methods of Technology Appraisal. Available from: <http://www.nice.org.uk/pdf/ TAP_Methods.pdf >. Acessado em: 21 maio. 2007.

Pitman R, Fisman D, Zaric GS, Postma M, Kretzschmar M, Edmunds J, et al. Dynamic transmission modeling: a report of the ISPOR-SMDM Modeling Good Research Practices Task Force--5. Value Health. 2012;15(6):828-34.

Ramsey SD, Willke RJ, Glick H, Reed SD, Augustovski F, Jonsson B, et al. Costeffectiveness analysis alongside clinical trials II-An ISPOR Good Research Practices Task Force report. Value Health. 2015;18(2):161-72.

Roberts M, Russell LB, Paltiel AD, Chambers M, McEwan P, Krahn M. Conceptualizing a model: a report of the ISPOR-SMDM Modeling Good Research Practices Task Force--2. Value Health. 2012;15(6):804-11.

Santos M, Cintra MA, Monteiro AL, Santos B, Gusmao-Filho F, Andrade MV, et al. Brazilian Valuation of EQ-5D-3L Health States: Results from a Saturation Study. Med Decis Making. 2015.

Sculpher MJ, Pang FS, Manca A, Drummond MF, Golder S, Urdahl H, et al. Generalisability in economic evaluation studies in healthcare: a review and case studies. Health Technol Assess. 2004;8(49):iii-iv, 1-192.

Siebert U, Alagoz O, Bayoumi AM, Jahn B, Owens DK, Cohen DJ, et al. Statetransition modeling: a report of the ISPOR-SMDM Modeling Good Research Practices Task Force--3. Value Health. 2012;15(6):812-20.

Stephens J, Handke B, Doshi J. International survey of methods used in health technology assessment (HTA): does practice meet the principles proposed for good research? Comp Eff Res. 2012;2:29-44.

Willke RJ. Tailor-made or off-the-rack? The problem of transferability of health economic data. Expert Rev Pharmacoecon Outcomes Res. 2003:3(1):1-4. 